\title{
HISTOPATHOLOGICAL PATTERNS OF SINONASAL MASSES: A HOSPITAL-BASED STUDY
}

\author{
Dharmakanta Kumbhakar ${ }^{1}$
}

${ }^{1}$ Associate Professor, Department of Pathology, Tezpur Medical College and Hospital, Tezpur, Sonitpur, Assam.

\section{ABSTRACT}

\section{BACKGROUND}

Sinonasal masses are one of the commonest cause of nasal obstruction with or without nasal discharge. Sinonasal masses may show diverse histopathological patterns. The sinonasal masses may be non-neoplastic lesions like nasal polyps either inflammatory or allergic type, traumatic lesions, granulomatous lesions or neoplastic lesions. Neoplasms of sinonasal masses form a wide spectrum like angiofibroma, capillary haemangioma at one end while squamous cell carcinoma at the other end of the spectrum. Benign neoplastic lesions arising from nasal cavities, paranasal sinuses and nasopharynx may threaten the patients because of their high recurrence rate and propensity for malignant potential. Any masses, which are seen in the area of nasal cavities and paranasal sinuses are termed as "sinonasal masses." Histopathological examination is the only means of determining the nature and patterns of these sinonasal masses, i.e. non-neoplastic versus neoplastic.

\section{AIMS AND OBJECTIVES}

A hospital-based study to determine the histopathological patterns of sinonasal masses.

\section{MATERIALS AND METHODS}

The present study was based on histopathological analysis of 120 specimens of sinonasal masses presented at Pathology Department of Tezpur Medical College and Hospital, Tezpur, Assam, for histopathological examination in the period of two years from June 1, 2014, to May 31, 2016.

\section{RESULTS AND OBSERVATIONS}

A total of 120 patients of sinonasal masses of all age groups with M:F ratio of 1.61:1 were included in the study. Majority of the patients with sinonasal masses were in the age group of 11-20 years. Out of the total 120 studied sinonasal masses, 83 sinonasal masses (69.17\%) were non-neoplastic lesions and 37 (30.83\%) sinonasal masses were neoplastic lesions with a non-neoplastic and neoplastic ratio of $2.24: 1$. All the $83(69.17 \%)$ non-neoplastic masses were nasal polyps. Out of the neoplastic masses, 33 $(27.50 \%)$ sinonasal masses were benign tumours and $04(3.33 \%)$ sinonasal masses were malignant tumours. Among 37 total neoplastic masses, $33(89.19 \%)$ were benign tumours and $04(10.81 \%)$ were malignant tumours with a benign and malignant tumours ratio of 8.25:1. Angiofibroma of $18(15.00 \%)$ sinonasal masses were the commonest benign tumours detected. Squamous cell carcinomas were the most common malignant tumours. One case of adenoid cystic carcinoma and one case of mucosal melanoma was also detected amongst the studied sinonasal masses.

\section{DISCUSSION}

Sinonasal masses form a wide spectrum of lesions in the nasal cavities and paranasal sinuses ranging from non-neoplastic lesions like nasal polyps either inflammatory or allergic type to tumours like angiofibroma, capillary haemangioma at one end while squamous cell carcinoma at the other end of the spectrum. Histopathological examination is the only means of determining the nature and patterns of the sinonasal masses, i.e. non-neoplastic versus neoplastic. It is conclusive in diagnosing the exact polypoid lesions.

\section{CONCLUSION}

Sinonasal masses need histopathological examination for proper diagnosis and follow up of the patients. This study gave the histopathological patterns of sinonasal masses of the patients that attended our institute during the study period with different clinical presentations. As, it was a hospital-based study, it might not reflect the exact scenario of the population at large.

\section{KEYWORDS}

Sinonasal Mass, Nasal Polyp, Neoplastic, Non-neoplastic, Angiofibroma, Capillary haemangioma, Squamous Cell Carcinoma, Adenoid Cystic Carcinoma, Mucosal Melanoma.

HOW TO CITE THIS ARTICLE: Kumbhakar D. Histopathological patterns of sinonasal masses: a hospital-based study. J. Evolution Med. Dent. Sci. 2016;5(64):4564-4568, DOI: 10.14260/jemds/2016/1041

Financial or Other, Competing Interest: None.

Submission 21-06-2016, Peer Review 27-07-2016,

Acceptance 03-08-2016, Published 11-08-2016.

Corresponding Author:

Dr. Dharmakanta Kumbhakar,

Associate Professor,

Department of Pathology,

Tezpur Medical College and Hospital,

Tezpur, Sonitpur

Assam.

E-mail: drkdharmakanta@yahoo.com

DOI: $10.14260 /$ jemds/2016/1041

\section{INTRODUCTION}

The nasal cavities, paranasal sinuses and nasopharynx form a complex functional unit. The two main types of epithelia that cover these structures are stratified squamous epithelium and respiratory-type pseudostratified columnar epithelium. The pathological processes that involve this region reflect some commonality, particularly in cases of nasal cavities and paranasal sinuses.[1] Any masses, which are seen in the area of nasal cavities and paranasal sinuses are termed as "sinonasal masses." Nasal obstruction with or without nasal discharge is one of the commonest clinical presentation of the sinonasal 
masses. Sinonasal masses are found in almost all age groups of people.

The sinonasal masses may be non-neoplastic lesions like nasal polyps either inflammatory or allergic type, traumatic lesions, granulomatous lesions or neoplastic lesions. ${ }^{[2]}$ As per authors like Humayun et al (2010),[3] A. Lathi et al (2011)[4] and Modh SK et al (2013) ${ }^{[5]}$ nasal polyps are the most common sinonasal masses, which are characterised by bulging of oedematous mucosal connective tissues covered by respiratory epithelium. The nasal polyps are not true tumours and their formation is associated with inflammation, allergy, or mucoviscidosis. ${ }^{[6,7]}$ Recurrent attacks of rhinitis eventually lead to nasal polyps formation, which may reach 3 to $4 \mathrm{~cm}$ in length.[8] Clinically, they appear as soft polypoid masses that extend laterally from the mucosa into the anterior part of the middle meatus. ${ }^{[9]}$ Bilaterality is the rule. On histology, these polyps consist of oedematous mucosa having a loose stroma, often harbouring hyperplastic or cystic mucous glands and infiltrated with varieties of inflammatory cells including predominantly neutrophils, eosinophils and plasma cells with occasional clusters of lymphocytes.

Sinonasal neoplasms may be benign or malignant. Papilloma, capillary haemangioma and angiofibroma are commonly found benign sinonasal neoplastic lesions.

Nasal angiofibroma is a highly vascular benign tumour of adolescent male with episodes of epistaxis and nasal obstruction as clinical presentations. Inverted papilloma is a locally invasive benign tumour of this region.[10] As the name implies, the papillomatous proliferation of squamous epithelium instead of producing an exophytic growth extends into the mucosa, that is inverted. Capillary haemangioma is another common benign tumour of this region. Malignant tumours in the nasal cavities and paranasal sinuses account for less than $1 \%$ of all carcinomas and for about $3 \%$ of the neoplasms of the head and neck region. Squamous cell carcinoma is the most common microscopic type of sinonasal neoplasm affecting the nasal skin and nasal cavities.[11] On histologic examination, the keratinising and non-keratinising squamous cell carcinomas more or less resemble usual welldifferentiated and poorly-differentiated squamous cell carcinomas arising in other locations. Humayun et al (2010) ${ }^{[3]}$ in their study reported an incidence of squamous cell carcinomas $41.67 \%$ (5 out of 12 ) among malignant sinonasal masses with average age at diagnosis of 51 years and male predominance. Adenocarcinomas of various types comprise $10 \%$ to $20 \%$ of all primary malignant neoplasms of the nasal cavities and paranasal sinuses.[12] Adenoid cystic carcinomas also occur in the maxillary sinuses and nasal cavities. Mucosal melanomas of the head and neck account for less than $1 \%$ of the lesions in this site.[13] Their most common locations are the nasal cavities followed by antrum, ethmoid and frontal sinuses. They arise from melanocytes located in the epithelium and stroma of the respiratory mucosa. Considering the importance of the sinonasal masses, a hospital-based study was undertaken with the aims and objectives to determine the histopathological patterns of the sinonasal masses in the Pathology Department of Tezpur Medical College and Hospital, Tezpur, Assam, for a period of two (02) years from June 1, 2014 to May 31, 2016.

\section{MATERIALS AND METHODS}

The study was carried out on 120 specimens of sinonasal masses presented at Pathology Department of Tezpur Medical College and Hospital, Tezpur, Assam, for histopathological examination in the period of two years from June 1, 2014 to May 31, 2016. Patients of all age groups and both sexes with sinonasal masses that attended our institute during the study period were included in the study. Data regarding their clinical profile was collected from the otorhinolaryngology department of Tezpur Medical College and Hospital, Tezpur, Assam.

A total of 120 specimens of sinonasal masses underwent biopsy interpretation. The specimens collected for the study were sent by the Otorhinolaryngology Department of the institute fixed in 10\% formalin. The specimens were observed grossly in the Pathology Department and findings were noted in the note sheets. The biopsy tissues were then sectioned and processed in the conventional manner as described in the "Theory and practice of histological techniques", $5^{\text {th }}$ edition, 2002, edited by J D Bancroft and Marilyn Gamble.[14] After completion of processing, they were made in paraffin blocks and cut in rotatory microtome of about $3-5 \mu$ thickness. The sections were stained by conventional Haematoxylin and Eosin staining mounted in DPX and examined under microscope. Their special features were noted and a histopathological diagnosis of the sinonasal masses were done.

\section{RESULTS AND OBSERVATIONS}

The study included a total of 120 specimens of sinonasal masses for histopathological evaluation.

The age of the patients with sinonasal masses included in the study ranged from 5 to 60 years with a mean age of 33 years. Majority of the patients with sinonasal masses in the study were in the age group of 11-20 yrs. (Cite Table-1). There were $74(61.67 \%)$ males and $46(38.33 \%)$ females with M:F ratio of 1.61:1 (Cite Fig.1).

The common clinical presentations of the sinonasal masses were - nasal obstruction in 117 cases of sinonasal masses (97.50\%), rhinorrhea in 59 cases of sinonasal masses (49.17\%), hyposmia in 38 cases of sinonasal masses $(31.67 \%)$ and headache in 20 cases of sinonasal masses (16.67\%). Epistaxis was noticed in 22 cases of sinonasal masses (18.33\%) (Cite Table-2). Sinonasal masses were bilateral in 26 cases $(21.67 \%)$, left sided in 49 cases $(40.83 \%)$ and right sided in 45 cases (37.50\%) (Cite Figure-2).

According to Table-3, out of the total 120 sinonasal masses, 83 sinonasal masses were non-neoplastic lesions $(69.17 \%)$ and 37 sinonasal masses were neoplastic lesions (30.83\%) with a non-neoplastic and neoplastic ratio of 2.24:1. (Cite Figure-3). All the 83 (69.17\%) non-neoplastic masses were nasal polyps. Out of the neoplastic masses, $33(27.50 \%)$ sinonasal masses were benign tumours and 04 (3.33\%) sinonasal masses were malignant tumours. (Cite Figure-4). Among 37 total neoplastic masses, 33 (89.19\%) were benign tumours and $04(10.81 \%)$ were malignant tumours with a benign and malignant tumours ratio of 8.25:1 (Cite Figure-5). Among the benign neoplastic masses, angiofibromas $(15.00 \%)$ were the commonest benign tumours encountered in the present study followed by capillary haemangiomas $(6.67 \%)$. Among the malignant sinonasal masses, squamous cell carcinomas (Cite Figure-6 and 7) were the commonest malignant tumours. One case each of adenoid cystic carcinoma (Cite Figure-8) and mucosal melanoma was also detected during the study period. 


\begin{tabular}{|c|c|c|}
\hline Age Range & $\begin{array}{c}\text { Number of Cases } \\
\text { (n=120) }\end{array}$ & $\begin{array}{c}\text { Percentage } \\
\text { (\%) }\end{array}$ \\
\hline $1-10$ Yrs. & 12 & $10.00 \%$ \\
\hline $11-20$ Yrs. & 43 & $35.83 \%$ \\
\hline $21-30$ Yrs. & 30 & $25.00 \%$ \\
\hline $31-40$ Yrs. & 24 & $20.00 \%$ \\
\hline $41-50$ Yrs. & 06 & $5.00 \%$ \\
\hline 51-60 Yrs. & 05 & $4.17 \%$ \\
\hline \multicolumn{2}{|c|}{ Table 1: Age Distribution of Patients Presented } \\
\hline \multicolumn{2}{|c|}{ with Sinonasal Masses. } \\
\hline
\end{tabular}

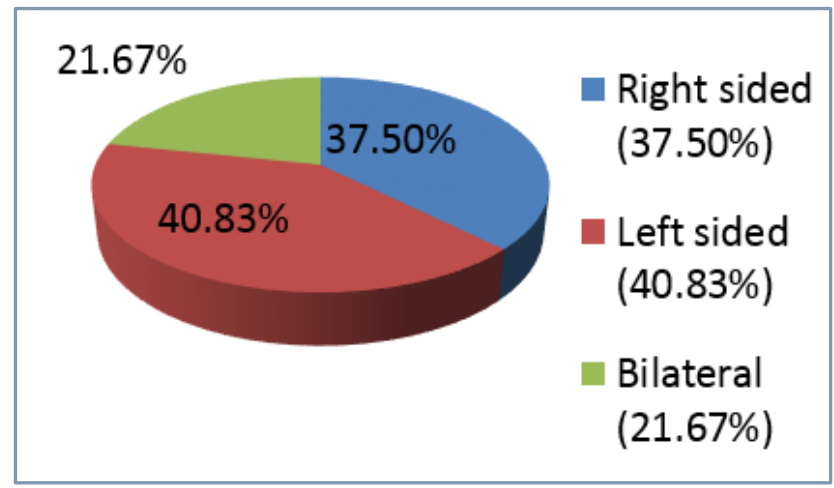

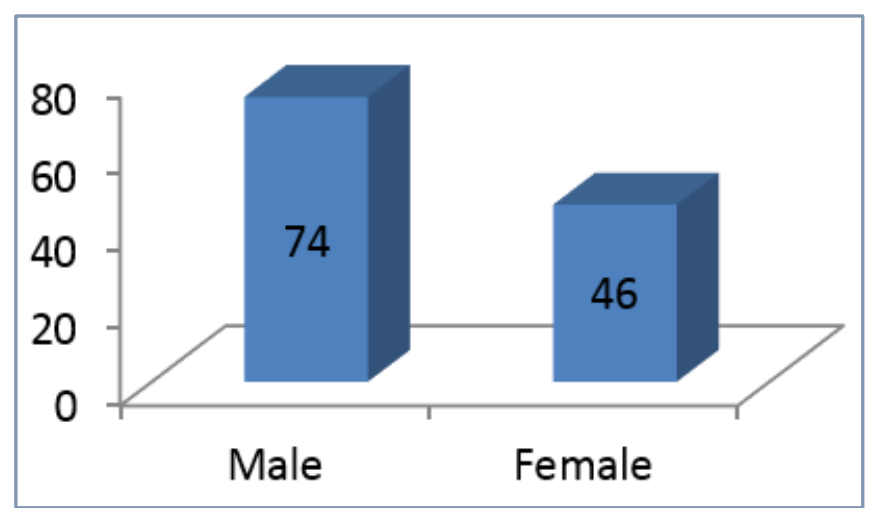

Fig. 1: Sex Distribution of Patients Producing Sinonasal Masses (Numbers)

\begin{tabular}{|c|c|c|}
\hline Clinical Presentation & Numbers & Percentage \\
\hline Nasal obstruction & 117 & $97.50 \%$ \\
\hline Rhinorrhea & 59 & $49.17 \%$ \\
\hline Hyposmia & 38 & $31.67 \%$ \\
\hline Headache & 20 & $16.67 \%$ \\
\hline Epistaxis & 22 & $18.33 \%$ \\
\hline Table 2: Common Clinical Presentations of \\
Sinonasal Masses \\
\hline
\end{tabular}

\begin{tabular}{|c|c|c|}
\hline $\begin{array}{c}\text { Diseases Producing } \\
\text { Sinonasal Masses }\end{array}$ & $\begin{array}{l}\text { Number } \\
\text { of Cases } \\
(n=120)\end{array}$ & $\begin{array}{c}\text { Percentage } \\
\text { (\%) }\end{array}$ \\
\hline A).Non-neoplastic & 83 & $69.17 \%$ \\
\hline 1) Polyp & 83 & $69.17 \%$ \\
\hline B) Neoplastic & 37 & $30.83 \%$ \\
\hline 1). Benign & 33 & $27.50 \%$ \\
\hline i) Angiofibroma & 18 & $15.00 \%$ \\
\hline ii) Capillary haemangioma & 08 & $6.67 \%$ \\
\hline iii) Inverted papilloma & 06 & $5.00 \%$ \\
\hline iv) Neurofibroma & 01 & $0.83 \%$ \\
\hline 2). Malignant & 04 & $3.33 \%$ \\
\hline i) Squamous cell carcinoma & 02 & $1.67 \%$ \\
\hline ii) Adenoid cystic carcinoma & 01 & $0.83 \%$ \\
\hline iii) Mucosal melanoma & 01 & $0.83 \%$ \\
\hline \multicolumn{3}{|c|}{$\begin{array}{l}\text { Table 3: Biopsy Interpretation of } \\
\text { Sinonasal Masses }(n=120)\end{array}$} \\
\hline
\end{tabular}

Fig. 2: Site Distribution of Sinonasal Mass (Percentage)

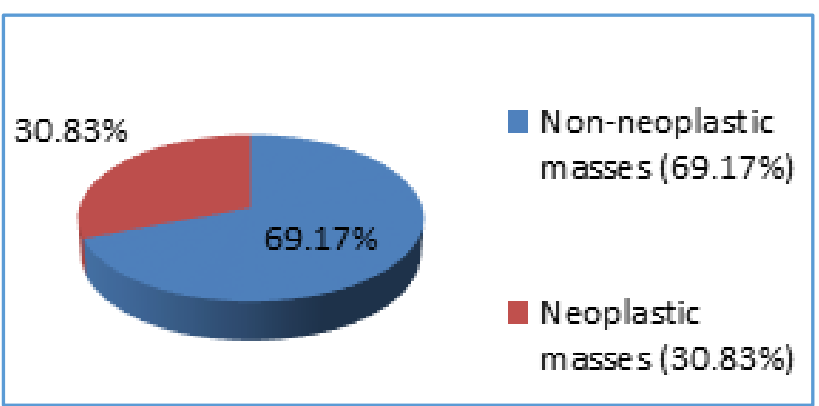

Fig. 3: Percentage of Non-Neoplastic and Neoplastic Sinonasal Masses

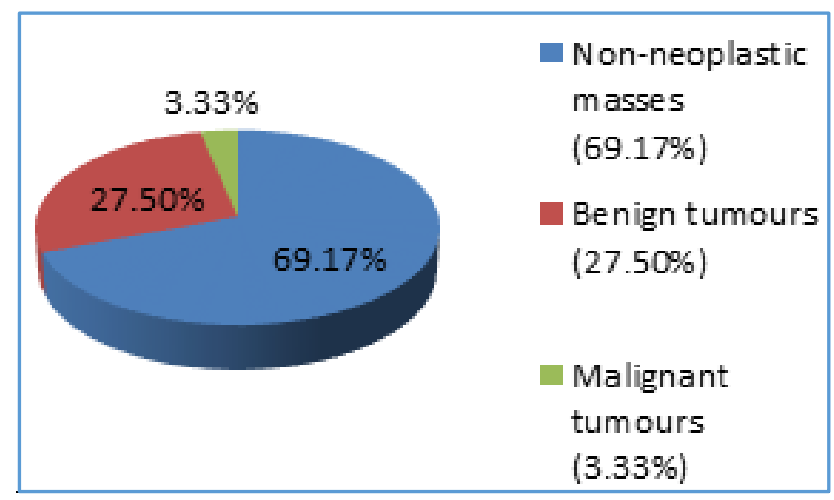

Fig. 4: Pathology of Sinonasal Masses According to Histopathological Patterns (Percentage).

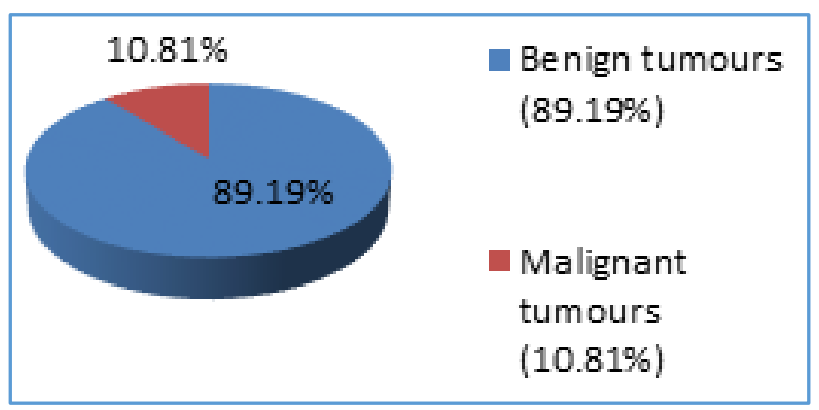

Fig. 5: Percentage of Benign and Malignant Tumours amongst Neoplastic Sinonasal Masses 


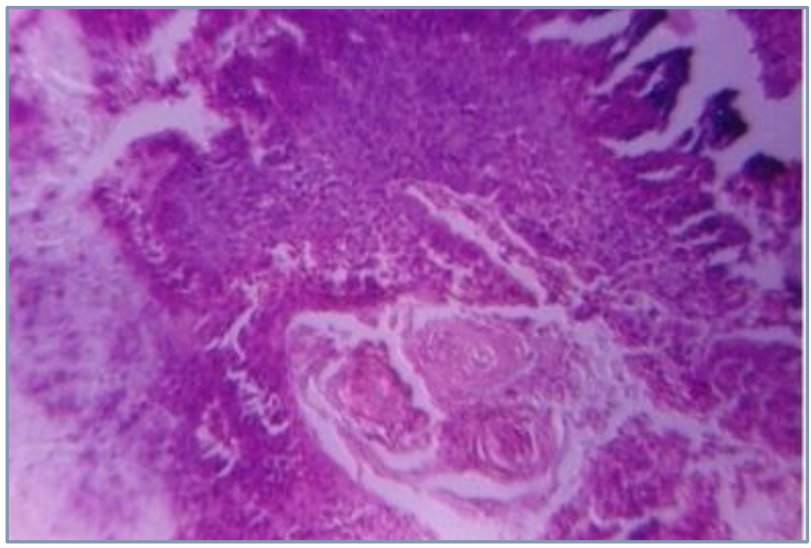

Fig. 6: Photomicrograph Showing HPE from Squamous Cell Carcinoma of Sinonasal Masses. H and E Stain (Low Power View)

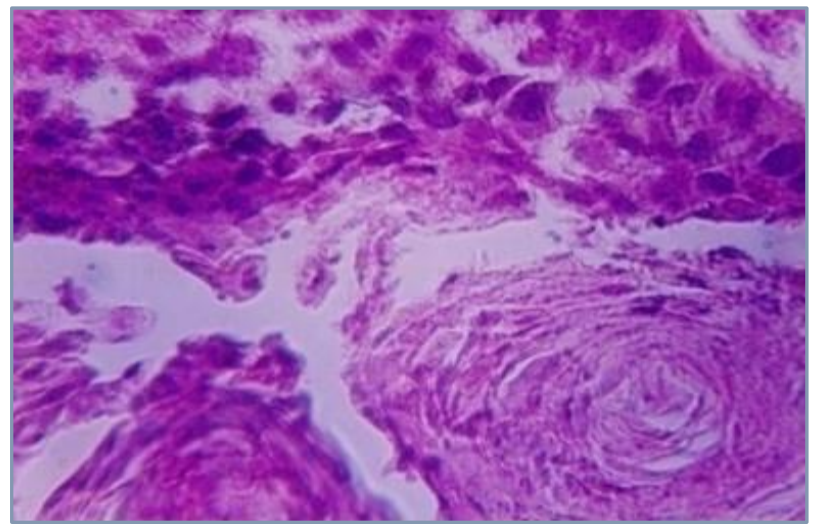

Fig. 7: Photomicrograph Showing HPE from Squamous Cell Carcinoma of Sinonasal Masses. H and E Stain (High Power View)

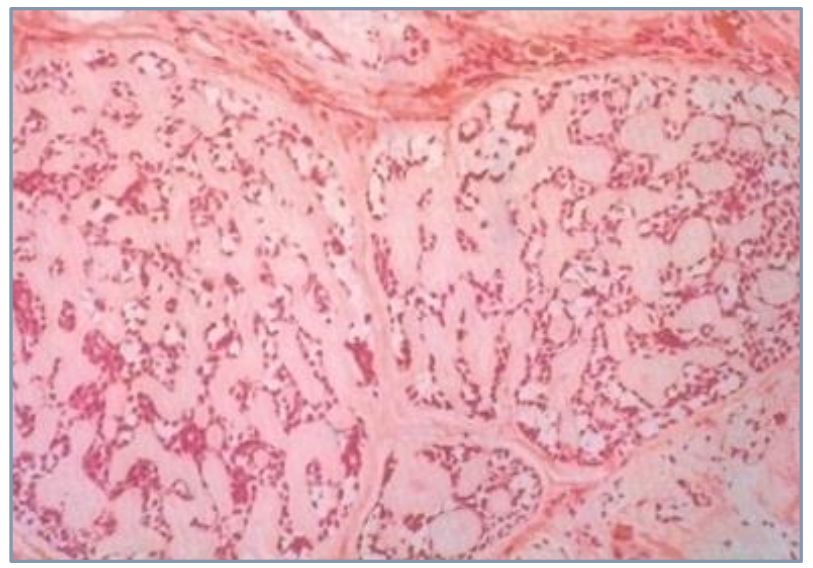

Fig. 8: Photomicrograph Showing HPE from Adenoid Cystic Carcinoma of Sinonasal Masses. H and E Stain (High Power View)

\section{DISCUSSION}

Sinonasal masses are one of the commonest cause of nasal obstruction with or without nasal discharge. The present study revealed that both male and female patients with $\mathrm{M}: \mathrm{F}$ ratio of 1.61:1 of all age groups ranged from 5 to $60 \mathrm{yrs}$. with a mean age of 33 years with majority of the patients of age group 11-20 years presented sinonasal masses bilaterally in $26(21.67 \%)$, left sided in $49(40.83 \%)$ and right sided in 45 (37.50\%) patients with clinical presentations of nasal obstruction $(97.50 \%)$, rhinorrhea $(49.17 \%)$, hyposmia (31.67\%), headache (16.67\%) and epistaxis (18.33\%).
The sinonasal masses may be non-neoplastic lesions like nasal polyps either inflammatory or allergic type, traumatic lesions, granulomatous lesions or neoplastic lesions. Neoplasms form a wide spectrum like angiofibroma, capillary haemangioma at one end while squamous cell carcinoma at the other end of the spectrum. The present study revealed that nasal polyps (83 cases-69.17\%) were the commonest sinonasal masses similar to the findings of other authors like Humayun et al 2010,[3] A. Lathi et al 2011[4] and Modh SK et al 2013.[5] Among the benign neoplastic sinonasal masses, angiofibromas (18 cases-15.00\%) were the commonest followed by capillary haemangiomas ( 8 cases-6.67\%). Nasal angiofibroma is a benign tumour of adolescent male with episodes of epistaxis and nasal obstruction as clinical presentations. Similar pictures were reflected in the previous studies too. Epistaxis was the commonest clinical presentation in all cases of capillary haemangioma $(100 \%)$ followed by nasal obstruction (80\%) and mass effect in nasal cavities $(60 \%)$ of the cases. In the present study, 6 cases of inverted papillomas were found to produce masses in the sinonasal region constituting $5.00 \%$ of the cases. Two cases of squamous cell carcinoma were detected in the present study. All these squamous cell carcinoma cases belonged to male sex and in 51-60 years age group.

They presented with rapid onset sinonasal masses with nasal discharge and occasional bouts of bleeding per nose. Humayun et al 2010[3] in their study reported an incidence of $41.67 \%$ (5 out of 12) squamous cell carcinomas among malignant masses with average age at diagnosis of 51 years and male predominance. Svane-Knudsen et al 1998[15] have similarly reported squamous cell carcinoma to be the most commonly encountered malignancy of sinonasal tract in Denmark. The present study also detected squamous cell carcinomas to be the commonest with incidence of $50 \%$ (2 out of 4) among malignant sinonasal masses with male predominance in the age group of 51-60 years. Similar findings were reported in studies by Modh SK et al 2013.[5]

The present study revealed a single case of adenoid cystic carcinoma occurring in a 38-year-old male patient. He presented clinically with nasal obstruction and epistaxis for 3 months. According to Damjanov and Linder[11] adenoid cystic carcinomas account for $3-10 \%$ of all salivary gland neoplasms and most cases occur in the fourth to sixth decades of life. It also accounts for $5-15 \%$ of all malignant paranasal neoplasms. The present study also detected a single case of mucosal melanoma occurring in a male patient of age 47 years. According to Narasimhan K et al (2009) ${ }^{[13]}$ mucosal melanomas form roughly $4 \%$ of head and neck melanoma cases and comprise $3.5 \%$ of all malignancies in the sinonasal region. ${ }^{[4]}$ Leena Jain in her study reported a case of mucosal melanoma of the sinonasal region in a male patient of 45 years.

\section{CONCLUSION}

Sinonasal masses form a wide spectrum of lesions ranging from non-neoplastic lesions like nasal polyps either inflammatory or allergic type to tumours like angiofibroma, capillary haemangioma at one end while squamous cell carcinoma at the other end of the spectrum. Sinonasal masses need histopathological examination for proper diagnosis and follow up of the patients. Histopathological examination is the only means of determining the nature of the sinonasal 
masses, i.e. non-neoplastic versus neoplastic. It is conclusive in diagnosing the exact polypoid lesions. The study gave the histopathological patterns of sinonasal masses of the patients that attended our institute during the study period with different clinical presentations. Rare tumour like mucosal melanoma was even detected in the study. As, it is a hospitalbased study, it might not reflect the exact scenario of the population at large.

\section{ACKNOWLEDGEMENTS}

The author acknowledges the help and co-operation received from the faculties of ENT Dept., Tezpur Medical College, Tezpur, Assam, and all the technicians of Histopathology Section, Pathology Dept., Tezpur Medical College, Tezpur, Assam, while conducting the present study.

\section{REFERENCES}

1. Juan Rosai. Rosai and Ackerman's Surgical Pathology. Chapter 7. $10^{\text {th }}$ edn. vol. 1. Elsevier 2011:291-318.

2. Cotran RS, Kumar V, Collins T. Robbins, pathologic basis of disease. $6^{\text {th }}$ edn. Philadelphia: WB Saunders Company 1999:762-764.

3. Humayun AHM, Zahurul Huq AHM, Ahmed SMT, et al. Clinicopathological study of sinonasal masses. Bangladesh J Otorhinolaryngol 2010;16(1):15-22.

4. Lathi A, Syed MMA, Kalakoti P, et al. Clinicopathological profile of sinonasal masses: a study from a tertiary care hospital of India. ACTA Otorhinolaryngol Ital 2011;31(6):372-7.
5. Modh SK, Delwadia KN, Gonsai RN. Histopathological spectrum of sinonasal masses-a study of 162 cases. Int J Cur Res Rev 2013;5(3):83-91.

6. Davidson A, Hellquist HB. The so-called allergic nasal polyp. ORL J Otorhinolaryngol Rela Spec 1993;55(1): 305.

7. MacArther CJ, Gliklick R, McGill TJ, et al. Sinus complications in mucopolysaccharidosis IH/S (HurlerScheie syndrome). Int J Paediatr Otorhinolaryngol 1993;26(1):79-87.

8. Slavin RG. Nasal polyps and sinusitis. JAMA 1997;278(22):1849-54.

9. Laesen PL, Tos M. Origin of nasal polyp. Laryngoscope 1991;101(3):305-12.

10. Oberman HA. Papillomas of the nose and paranasal sinuses. Am J ClinPathol 1964;42:245-258.

11. Damjanov I, Linder J. Anderson's pathology. $10^{\text {thedn. St. }}$ Louis, Mosby 1996.

12. Rousch GC. Epidemiolgy of cancer of the nose and paranasal sinuses: current concept. Head Neck Surg 1979;2(1):3-11.

13. Narasimhan K, Kucuk O, Ho-Sheng L, et al. Sinonasal mucosal melanoma: a 13-year experience at a single institution. Skull Base 2009;19(4):255-62.

14. John D. Bancroft and Marilyn gamble. Theory and practice of histological techniques. $5^{\text {th }}$ edn. Edinburgh: Churchill Livingstone 2002.

15. Svane-Knudsen V, Jørgensen KE, Hansen 0, et al. Cancer of the nasal cavity and paranasal sinuses: a series of 115 patients. Rhinology 1998;36(1):12-4. 